\title{
An Analysis of Informal Academic Exchange Based on the Emuch Net
}

\section{Bai Yu}

Co-Innovation Center for Educational Informatization and Balanced Development of Basic Education, Central China Normal of University, Wuhan, China

\section{Email address: \\ 651952800@qq.com}

\section{To cite this article:}

Bai Yu. An Analysis of Informal Academic Exchange Based on the Emuch Net. Science Discovery. Vol. 5, No. 6, 2017, pp. $426-433$. doi: 10.11648/j.sd.20170506.16

Received: September 24, 2017; Accepted: October 5, 2017; Published: November 21, 2017

\begin{abstract}
The rapid development of information technology of today, informal academic exchanges are increasingly attracted people's attention, non-academic communication refers to the information producers and users directly between the exchange, do not need to rely on scientific literature to communicate the exchange. How to increase the exchange of students and enhance the academic level through the use of informal communication is a topic that we need to study. In this paper, we find the social network analysis of the research area of the Emuch Net research and living area, and study the characteristics of the interaction behavior of the researchers in the work forum, explore the communication situation of the insects, discover the problems and provide some suggestions for the follow-up development The reference value.
\end{abstract}

Keywords: Informal Academic Communication, Social Network Analysis, Forum

\section{基于小木虫论坛的非正式学术交流分析}

\section{白玉}

信息化与基础教育均衡发展协同创新中心，华中师范大学，武汉，中国

\section{邮箱}

651952800@qq.com

摘要：信息化飞速发展的今天，非正式学术交流目前越来越引起人们的关注，非正式学术交流是指信息生产者和利用者之 间直接进行的交流，不需要依靠科学文献进行传递的交流。如何通过利用非正式交流这个途径增加学生间的交流和增强学 术水平是目前亟待研究的一个话题。本文选取小木虫科研生活区的找工作板块进行了社会网络分析, 研究科研人员在找工 作论坛中的交互行为特点, 探索木虫们的交流情况, 发现其中的问题, 为后续的发展研究提供一定的参考价值。

关键词: 非正式学术交流, 社会网络分析, 论坛

\section{1. 网络论坛中的非正式学术交流}

\section{1. 非正式学术交流的概念及地位}

科学信息交流过程是指从科学信息的发布者到信息 用户之间各个环节的信息工作流程[1]。前苏联著名情报学
家米哈依洛夫根据科学信息交流是否依靠科学文献将其 划分为“正式交流过程”和“非正式交流过程”，其中，借助 科技文献进行科学信息交流的过程称为“正式交流过程”, 信息生产者和利用者之间直接进行的交流称为“非正式交 流过程”。 
作为科学信息交流的一个重要组成部分, 非正式交流 曾经在科学发展中起到十分巨大的作用。R. W. Swanson 所引用的调查结果表明, 被询问的1523名科学家和工程师 中通过非正式渠道获得的科学情报占全部科学情报的 $53 \%$ [2], 著名的情报学家普莱斯认为: “那些激励每位科 学家并作为其研究成为可能的信息中心, $80 \%$ 左右是他在 那些资料正式报道之前通过直接交流渠道从其他研究人 员那里得到的。”科学家戴安娜·克兰也对非正式交流给出 过高度的评价, 认为通过个人传播的思想的扩散要比仅通 过出版物扩散更有效, 缺乏交流与互动的领域, 知识增长 缓慢 [3]。因此, 非正式学术交流既是知识扩散的重要途径, 又是知识创新的重要源泉。虽然人们普遍承认非正式交流 的作用, 但事实上在以印刷物为核心的出版物体系形成之 后, 图书情报学界对非正式交流的作用和地位始终评价不 高, 尤其在17世纪中期科学期刊产生之后, 非正式交流在 科学交流体系中所起到的作用处于一个相对的低潮期[4]。

\section{2. 网络论坛环境下的非正式学术交流}

随着网络技术的不断进步, 信息在快速的更新, 文献 数量呈现指数型的增长趋势。对于这个大学生极度增长的 年代, 研究生和博士生也在不断增多, 找工作越来越成为 众多学生的人生难题。何去何从, 仅仅通过与自己老师和 同伴的交流是远远不够的, 同时建立在文献基础上的科学 交流体系因获取信息的滞后性和高成本, 以及在确保科学 信息自由、充分发布上的固有缺陷, 越来越不能满足科研 人员的交流需要。为了保证科学信息交流的及时通畅, 确 保科研人员的及时和顺利就业, 人们更加重视通过人际关 系渠道进行非正式的交流和沟通, 弥补身边现有资源的不 足。

在网络时代, 大多数的科研人员使用网络进行交流, 出现了包括电子邮件、博客圈、讨论组等在内的传统非正 式学术交流在网络环境下的延伸。伴随着交流手段的变化, 非正式学术交流的特点也发生了显著的变化。

\section{2. 社会网络分析方法的引入}

社会网络是指社会个体成员之间因为互动而形成的 相对稳定的关系体系, 社会网络关注的是人们之间的互动 和联系。非正式学术交流知识网络在本质上是一个由节点 和联系构成的社会网络, 因此可以借助社会网络分析方法 对非正式学术知识网络能力进行评价。

社会网络分析中, 网络密度是指网络中各个成员之间 联系的紧密度, 可以通过网络中实际存在的关系数与理论 上可能存在的关系数相比得到, 成员之间的联系越多, 该 网络的密度越大。中心性是度量整个网络中心化程度的重 要指标, 可分为点度中心度、接近中心度和中间中心度, 其中点度中心度指在社会网络中, 一个行动者与其他很多 行动者有直接联系, 该行动者就处在中心地位, 是最有社 会地位和最有权力的人; 中间中心度指如果一个行动者处 在许多交往网络的路径上, 可以认为此人处于重要地位, 具有控制他人交往的能力, 因为他人的交往需要通过该人 才能进行。凝聚子群指小团体, 团体中的一小群人关系很
紧密, 以至于结合形成一个次级团体, 它反映了非正式交 流的派系状况。

\section{3. 社会网络分析软件}

社会网络分析的软件有很多, 其中UCINET是研究者 较为常用的, 它是由加州大学欧文分校的一群网络分析者 编写的, 由于可以免费试用所以是目前使用最频繁的。本 文将就本软件的使用进行内容的分析。

\section{4. 小木虫找工作论坛交流特点的社会网络分析}

小木虫论坛(http://emuch.net/bbs/)成立于 2001 年 3 月 1 日, 是一个独立、纯学术、非经营性的免费个人论坛。一 直致力于打造国内学术前沿站点, 为中国学术研究提供免 费动力, 倡导学术的交流与共享 [5]。小木虫论坛拥有会员 近 30 万, 日访问量将近 30 万流量, 涵盖 10 个学科门类的专 业性学术科研交流合法论坛, 同样也是众多大学生、硕士 生和博士生交流找工作经验和困惑的大型交流平台。本文 拟以小木虫论坛为样本, 分析网络时代非正式学术交流的 特点。

\section{1. “小木虫论坛” 知识交流模式分析}

由于“小木虫”论坛是一个学术信息交流性质的综合 科研服务性网站, 全站非盈利、纯学术、独立的个人网站, 所以在这个论坛里进行知识交流的社区成员主要是拥有 较高的知识文化背景的人。这些会员主要来自国内各大院 校、科研院所的博硕士研究生、企业研发人员 [6]。小木虫 论坛里所有会员都拥有不同的级别, 同时具有不同头衔的 的成员在知识交流中扮演着不同的角色, 且都有自己的特 点。根据他们扮演的角色和特点可以分为以下四类群体:

一是领袖, 论坛中领袖数量一般很少, 但是他们在论 坛中起着不可或缺的作用。领袖一般本身知识面很广, 专 业知识能力过硬, 发帖的频率和发帖数量相对于论坛中其 他成员来说很高, 最重要的是在论坛的交流和知识共享过 程中贡献率最高。论坛中交流区上方都会有一个“JOB-EPI 牛人榜”, 一般牛人榜上面的会员即是论坛领袖。他们乐 于主动提供相关领域的知识, 分享个人经验和心得, 帮助 社区其他成员学习知识, 积极对其他成员的问题提供看法 和建议，经常带动社区成员间进行经验分享 [7]。

二是呼应者, 这些论坛成员一般会根据自己的实际情 况在其他会员发表的帖子下发表一些自己的心情和看法 等, 但是发文的知识含量较低, 他们对于社区整体的知识 形成与共享没有起到多大的作用。

三是共享者, 这些群体处于论坛的中等地位。相对于 领袖, 他们一般是被动地为成员提供知识。他们在论坛里 面的表现主要是发表个人看法或经验或者转载他人的看 法或其它媒体等方面的信息。

四是汶览者, 除了上述三类群体, 还有浏览者, 他们 是论坛的不速之客。这类群体很少发帖子甚至不发帖子, 他们来小木虫论坛的主要目的可能只是为了查询和汶览 相关信息，对论坛不起任何作用并且没有贡献率。 


\section{2. “小木虫论坛” 社会网络结构的形式化表达}

\subsection{1. 数据来源及处理}

在此需要说明的是, 小木虫找工作论坛因数据量过大, 本文仅用八爪鱼采集器爬取小木虫找工作分论坛从近三 个月、5000个页面进行了分析。

\subsection{2. 节点和关系的提取原则}

笔者在进行爬取数据的时候考虑到一般活跃的会员 发帖数多回帖数也会相应的多，而且我们选取了 5000 个页
面, 如果每一页面发帖数加回帖数都统计的话, 工作量实 在太庞大, 最终我们统计的是 5000 个帖子的发帖人和发帖 时间。

\subsection{3. 节点发帖统计与分析}

统计后发现 5000 个帖子中一共发帖的会员数量是 3982 个, 笔者将只发过一次帖的会员作为分析无关项排除, 剩下的会员这段时间内的发帖数从 2 个到 52 个依次不等， 从中笔者又选取了这段时间内发帖数从 52 到 5 的 34 名会员 如表1所示:

表1 发帖数目分析。

\begin{tabular}{|c|c|}
\hline 发帖人 & 发帖数目 \\
\hline 382777361 & 52 \\
\hline sunkaier & 17 \\
\hline nnnnnnnmmnn & 12 \\
\hline 落叶纷飞fd & 11 \\
\hline t191877459 & 10 \\
\hline huagongfeihu & 8 \\
\hline huxd03 & 8 \\
\hline qazwh & 7 \\
\hline xulei0228 & 7 \\
\hline ycwyj & 7 \\
\hline 沉默的角落 & 7 \\
\hline 带我飞啊 & 7 \\
\hline 东海之滨 & 7 \\
\hline 疯狂的维纳斯 & 7 \\
\hline 洋洋123456 & 7 \\
\hline rym2002 & 6 \\
\hline supremetom & 6 \\
\hline xiaoyi0799 & 6 \\
\hline 小达达达 & 6 \\
\hline 小朋友爱祖国 & 6 \\
\hline 燕大侠 & 6 \\
\hline 2010132102 & 5 \\
\hline dropcatch & 5 \\
\hline sdfg_0012 & 5 \\
\hline xnjd000 & 5 \\
\hline yaxinda & 5 \\
\hline 尘封风风 & 5 \\
\hline 江南风雨2014 & 5 \\
\hline 空蒙的天空 & 5 \\
\hline 懒懒行空 & 5 \\
\hline 全球漂 & 5 \\
\hline 小吱吱球 & 5 \\
\hline 心高路远 & 5 \\
\hline
\end{tabular}

为了进一步明确上述 33 名会员之间的是否存在交互和关系，笔者统计了5000个帖子中他们彼此之间是否有交互， 若有标为 1 , 若无则标为 0 . 通过对节点间联系的图形化表达 (为了分析的直观、简化表达, 略去节点名称, 只显示节点 的次序）得到表2。 
表2 小木虫找工作论坛主要节点间联系.

\begin{tabular}{|c|c|c|c|c|c|c|c|c|c|c|c|c|c|c|c|c|c|c|c|c|c|c|c|c|c|c|c|c|c|c|c|c|c|}
\hline & 1 & 2 & 3 & 4 & 5 & 6 & 7 & 8 & 9 & 10 & 11 & 12 & 13 & 14 & 15 & 16 & 17 & 18 & 19 & 20 & 21 & 22 & 23 & 24 & 25 & 26 & 27 & 28 & 29 & 30 & 31 & 32 & 33 \\
\hline 1 & 0 & 1 & 0 & 0 & 0 & 1 & 0 & 0 & 0 & 1 & 0 & 0 & 0 & 0 & 0 & 0 & 1 & 0 & 0 & 0 & 0 & 0 & 0 & 0 & 0 & 0 & 0 & 0 & 0 & 0 & 0 & 0 & 0 \\
\hline 2 & 1 & 0 & 1 & 1 & 1 & 1 & 0 & 0 & 0 & 0 & 0 & 1 & 0 & 0 & 0 & 0 & 0 & 1 & 0 & 0 & 1 & 0 & 0 & 1 & 0 & 0 & 0 & 1 & 0 & 1 & 1 & 1 & 1 \\
\hline 3 & 0 & 1 & 0 & 0 & 0 & 0 & 0 & 0 & 0 & 0 & 0 & 0 & 0 & 0 & 0 & 1 & 0 & 0 & 0 & 0 & 0 & 0 & 0 & 0 & 0 & 1 & 0 & 0 & 0 & 1 & 0 & 0 & 0 \\
\hline 5 & 0 & 1 & 0 & 0 & 0 & 0 & 0 & 0 & 0 & 1 & 0 & 0 & 0 & 0 & 0 & 0 & 0 & 0 & 0 & 0 & 0 & 0 & 0 & 0 & 0 & 0 & 0 & 0 & 0 & 0 & 0 & 0 & 0 \\
\hline 6 & 1 & 1 & 0 & 0 & 0 & 0 & 0 & 0 & 0 & 0 & 0 & 0 & 0 & 0 & 0 & 0 & 0 & 0 & 0 & 0 & 0 & 0 & 0 & 0 & 0 & 0 & 0 & 1 & 0 & 0 & 0 & 0 & 0 \\
\hline 7 & 0 & 0 & 0 & 0 & 0 & 0 & 0 & 0 & 0 & 0 & 0 & 0 & 0 & 0 & 0 & 0 & 0 & 0 & 0 & 0 & 0 & 0 & 0 & 0 & 0 & 0 & 0 & 0 & 0 & 1 & 0 & 0 & 0 \\
\hline 8 & 0 & 0 & 0 & 0 & 0 & 0 & 0 & 0 & 0 & 0 & 0 & 0 & 0 & 0 & 0 & 0 & 0 & 0 & 0 & 0 & 1 & 0 & 0 & 0 & 0 & 0 & 0 & 0 & 0 & 0 & 0 & 0 & 0 \\
\hline 10 & 1 & 0 & 0 & 0 & 1 & 0 & 0 & 0 & 0 & 0 & 0 & 0 & 0 & 0 & 0 & 0 & 0 & 0 & 0 & 0 & 0 & 0 & 0 & 0 & 0 & 0 & 0 & 0 & 0 & 0 & 0 & 0 & 0 \\
\hline 11 & 0 & 0 & 0 & 0 & 0 & 0 & 0 & 0 & 0 & 0 & 0 & 0 & 1 & 0 & 0 & 0 & 0 & 0 & 0 & 0 & 0 & 0 & 0 & 0 & 0 & 0 & 0 & 0 & 0 & 0 & 0 & 0 & 0 \\
\hline 12 & 0 & 1 & 0 & 0 & 0 & 0 & 0 & 0 & 0 & 0 & 0 & 0 & 0 & 0 & 0 & 0 & 1 & 0 & 0 & 0 & 0 & 0 & 0 & 0 & 1 & 0 & 0 & 0 & 0 & 0 & 0 & 0 & 0 \\
\hline 13 & 0 & 0 & 0 & 0 & 0 & 0 & 0 & 0 & 0 & 0 & 1 & 0 & 0 & 0 & 0 & 0 & 0 & 0 & 0 & 0 & 0 & 0 & 0 & 0 & 0 & 0 & 0 & 0 & 0 & 0 & 0 & 0 & 0 \\
\hline 14 & 0 & 0 & 0 & 0 & 0 & 0 & 0 & 0 & 0 & 0 & 0 & 0 & 0 & 0 & 0 & 0 & 0 & 0 & 1 & 0 & 0 & 0 & 0 & 0 & 0 & 0 & 0 & 0 & 0 & 1 & 0 & 0 & 0 \\
\hline 15 & 0 & 0 & 0 & 0 & 0 & 0 & 0 & 0 & 0 & 0 & 0 & 0 & 0 & 0 & 0 & 0 & 0 & 1 & 0 & 0 & 0 & 0 & 0 & 0 & 0 & 0 & 0 & 0 & 0 & 0 & 0 & 0 & 0 \\
\hline 16 & 0 & 0 & 1 & 0 & 0 & 0 & 0 & 0 & 0 & 0 & 0 & 0 & 0 & 0 & 0 & 0 & 0 & 0 & 0 & 0 & 0 & 0 & 0 & 0 & 0 & 0 & 0 & 0 & 0 & 0 & 0 & 0 & 0 \\
\hline 17 & 1 & 1 & 0 & 0 & 0 & 0 & 0 & 0 & 0 & 0 & 0 & 1 & 0 & 0 & 0 & 0 & 0 & 0 & 0 & 0 & 0 & 0 & 0 & 0 & 0 & 0 & 0 & 0 & 0 & 0 & 0 & 0 & 0 \\
\hline 19 & 0 & 0 & 0 & 0 & 0 & 0 & 0 & 0 & 0 & 0 & 0 & 0 & 0 & 1 & 0 & 0 & 0 & 0 & 0 & 0 & 0 & 0 & 0 & 0 & 0 & 0 & 0 & 0 & 0 & 0 & 0 & 0 & 0 \\
\hline 20 & 0 & 0 & 0 & 0 & 0 & 0 & 0 & 0 & 0 & 0 & 0 & 0 & 0 & 0 & 0 & 0 & 0 & 0 & 0 & 0 & 0 & 0 & 0 & 0 & 0 & 0 & 1 & 0 & 0 & 0 & 0 & 0 & 0 \\
\hline 21 & 0 & 1 & 0 & 0 & 0 & 0 & 0 & 1 & 0 & 0 & 0 & 0 & 0 & 0 & 0 & 0 & 0 & 0 & 0 & 0 & 0 & 0 & 0 & 0 & 0 & 0 & 0 & 0 & 0 & 0 & 0 & 0 & 0 \\
\hline 22 & 0 & 0 & 0 & 0 & 0 & 0 & 0 & 0 & 0 & 0 & 0 & 0 & 0 & 0 & 0 & 0 & 0 & 0 & 0 & 0 & 0 & 0 & 0 & 0 & 0 & 0 & 0 & 0 & 0 & 0 & 0 & 0 & 0 \\
\hline 23 & 0 & 0 & 0 & 0 & 0 & 0 & 0 & 0 & 0 & 0 & 0 & 0 & 0 & 0 & 0 & 0 & 0 & 0 & 0 & 0 & 0 & 0 & 0 & 0 & 0 & 0 & 0 & 0 & 0 & 0 & 0 & 0 & 0 \\
\hline 24 & 0 & 1 & 0 & 1 & 0 & 0 & 0 & 0 & 0 & 0 & 0 & 0 & 0 & 0 & 0 & 0 & 0 & 0 & 0 & 0 & 0 & 0 & 0 & 0 & 0 & 0 & 0 & 0 & 0 & 0 & 0 & 1 & 0 \\
\hline 25 & 0 & 0 & 0 & 0 & 0 & 0 & 0 & 0 & 0 & 0 & 0 & 1 & 0 & 0 & 0 & 0 & 0 & 1 & 0 & 0 & 0 & 0 & 0 & 0 & 0 & 0 & 0 & 0 & 0 & 0 & 0 & 0 & 0 \\
\hline 26 & 0 & 0 & 1 & 0 & 0 & 0 & 0 & 0 & 0 & 0 & 0 & 0 & 0 & 0 & 0 & 0 & 0 & 0 & 0 & 0 & 0 & 0 & 0 & 0 & 0 & 0 & 0 & 0 & 0 & 0 & 0 & 0 & 0 \\
\hline 27 & 0 & 0 & 0 & 0 & 0 & 0 & 0 & 0 & 0 & 0 & 0 & 0 & 0 & 0 & 0 & 0 & 0 & 0 & 0 & 1 & 0 & 0 & 0 & 0 & 0 & 0 & 0 & 0 & 0 & 0 & 0 & 0 & 0 \\
\hline 28 & 0 & 1 & 0 & 0 & 0 & 1 & 0 & 0 & 0 & 0 & 0 & 0 & 0 & 0 & 0 & 0 & 0 & 0 & 0 & 0 & 0 & 0 & 0 & 0 & 0 & 0 & 0 & 0 & 0 & 0 & 0 & 0 & 0 \\
\hline 29 & 0 & 0 & 0 & 0 & 0 & 0 & 0 & 0 & 0 & 0 & 0 & 0 & 0 & 0 & 0 & 0 & 0 & 0 & 0 & 0 & 0 & 0 & 0 & 0 & 0 & 0 & 0 & 0 & 0 & 0 & 0 & 0 & 0 \\
\hline 30 & 0 & 1 & 1 & 0 & 0 & 0 & 1 & 0 & 0 & 0 & 0 & 0 & 0 & 1 & 0 & 0 & 0 & 0 & 0 & 0 & 0 & 0 & 0 & 0 & 0 & 0 & 0 & 0 & 0 & 0 & 0 & 0 & 0 \\
\hline 31 & 0 & 1 & 0 & 0 & 0 & 0 & 0 & 0 & 0 & 0 & 0 & 0 & 0 & 0 & 0 & 0 & 0 & 0 & 0 & 0 & 0 & 0 & 0 & 0 & 0 & 0 & 0 & 0 & 0 & 0 & 0 & 0 & 0 \\
\hline 32 & 0 & 1 & 0 & 0 & 0 & 0 & 0 & 0 & 0 & 0 & 0 & 0 & 0 & 0 & 0 & 0 & 0 & 0 & 0 & 0 & 0 & 0 & 0 & 0 & 0 & 0 & 0 & 0 & 0 & 0 & 0 & 0 & 0 \\
\hline 33 & 0 & 1 & 0 & 0 & 0 & 0 & 0 & 0 & 0 & 0 & 0 & 0 & 0 & 0 & 0 & 0 & 0 & 0 & 0 & 0 & 0 & 0 & 0 & 0 & 0 & 0 & 0 & 0 & 0 & 0 & 0 & 0 & 0 \\
\hline
\end{tabular}

\section{3. 小木虫找工作论坛的社会网络结构分析}

笔者将表 2 中 33 个主要节点的关系图数值表格导入 UCINET社会网络分析软件中, 最终可以计算出小木虫找 工作论坛中密度、节点中心度、接近中心度、中间中心度 和凝聚子群等分析。

\section{3. 1. 模态和层次分析}

社会网络有多种类型, 划分的根据可以有多个, 例如 可以根据行动者集合的性质, 这就涉及到“模”这个概念。 模指的是行动者的集合, 社会网络分析中所说的“行动者” 可以是任何一个社会单位或者社会实体。模的数目指的是 网络中社会行动者集合的类型的数目。如果研究对象仅仅
是一个集合的行动者, 研究行动者集合内部各个行动者之 间的关系, 这种网络叫做1-模网络。依此类推, 2-模网络 研究两类行动者群体之间的关系, 或者一类行动者和一类 事件之间的关系[8]。小木虫找工作分论坛的会员是一个集 合的行动者, 因此属于1-模网络。

\section{3. 2 . 密度分析}

整体网络密度计算方法为: “实际存在的关系总数”除 以“理论上最多可能存在的关系总数”, 实际上等于所有可 能存在的线的平均值。小木虫找工作论坛交流密度为 0.0644（图1）。

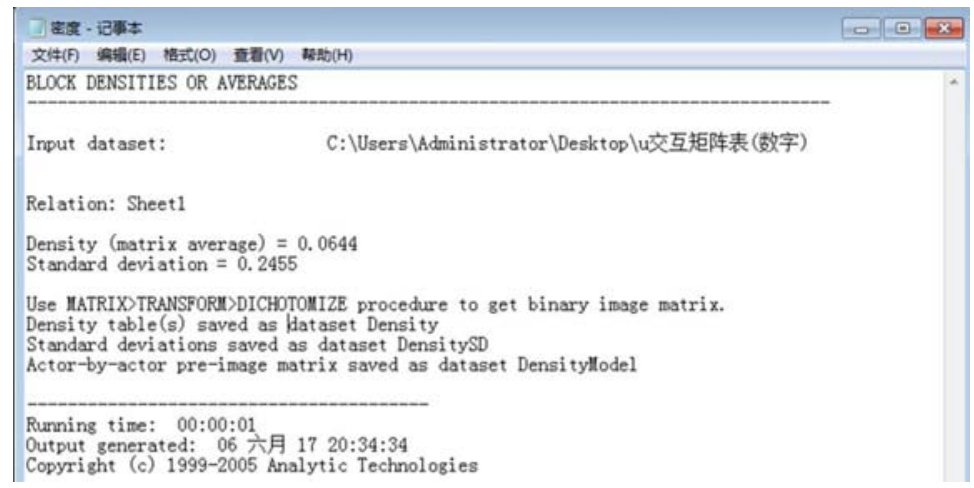

图1 小木虫找工作论坛的交流密度. 


\subsection{3. 中心性分析}

(1) 结点中心性

首先笔者分析了论坛中每个个体的结点中心度（表3），即该结点与其他结点相连接的程度。

表3 小木虫找工作论坛结点中心性状况。

\begin{tabular}{|c|c|c|c|c|}
\hline Serial Number & Number & Degree & NrmDegree & Share \\
\hline 1 & 2 & 15.000 & 46.875 & 0.214 \\
\hline 2 & 1 & 4.000 & 12.500 & 0.057 \\
\hline 3 & 3 & 4.000 & 12.500 & 0.057 \\
\hline 4 & 30 & 4.000 & 12.500 & 0.057 \\
\hline 5 & 17 & 3.000 & 9.375 & 0.043 \\
\hline 6 & 12 & 3.000 & 9.375 & 0.043 \\
\hline 7 & 24 & 3.000 & 9.375 & 0.043 \\
\hline 8 & 18 & 3.000 & 9.375 & 0.043 \\
\hline 9 & 6 & 3.000 & 9.375 & 0.043 \\
\hline 10 & 5 & 2.000 & 6.250 & 0.029 \\
\hline 11 & 21 & 2.000 & 6.250 & 0.029 \\
\hline 12 & 25 & 2.000 & 6.250 & 0.029 \\
\hline 13 & 4 & 2.000 & 6.250 & 0.029 \\
\hline 14 & 32 & 2.000 & 6.250 & 0.029 \\
\hline 15 & 10 & 2.000 & 6.250 & 0.029 \\
\hline 16 & 28 & 2.000 & 6.250 & 0.029 \\
\hline 17 & 14 & 2.000 & 6.250 & 0.029 \\
\hline 18 & 7 & 1.000 & 3.125 & 0.014 \\
\hline 19 & 11 & 1.000 & 3.125 & 0.014 \\
\hline 20 & 15 & 1.000 & 3.125 & 0.014 \\
\hline 21 & 19 & 1.000 & 3.125 & 0.014 \\
\hline 22 & 13 & 1.000 & 3.125 & 0.014 \\
\hline 23 & 31 & 1.000 & 3.125 & 0.014 \\
\hline 24 & 8 & 1.000 & 3.125 & 0.014 \\
\hline 25 & 33 & 1.000 & 3.125 & 0.014 \\
\hline 26 & 26 & 1.000 & 3.125 & 0.014 \\
\hline 27 & 27 & 1.000 & 3.125 & 0.014 \\
\hline 28 & 20 & 1.000 & 3.125 & 0.014 \\
\hline 29 & 16 & 1.000 & 3.125 & 0.014 \\
\hline 30 & 29 & 0.000 & 0.000 & 0.000 \\
\hline 31 & 23 & 0.000 & 0.000 & 0.000 \\
\hline 32 & 22 & 0.000 & 0.000 & 0.000 \\
\hline 33 & 9 & 0.000 & 0.000 & 0.000 \\
\hline
\end{tabular}

为了更加清晰的明确每位会员在整个论坛中的地位, 笔者又用UCINET软件将关联图可视化出来, 如图 2 所示:

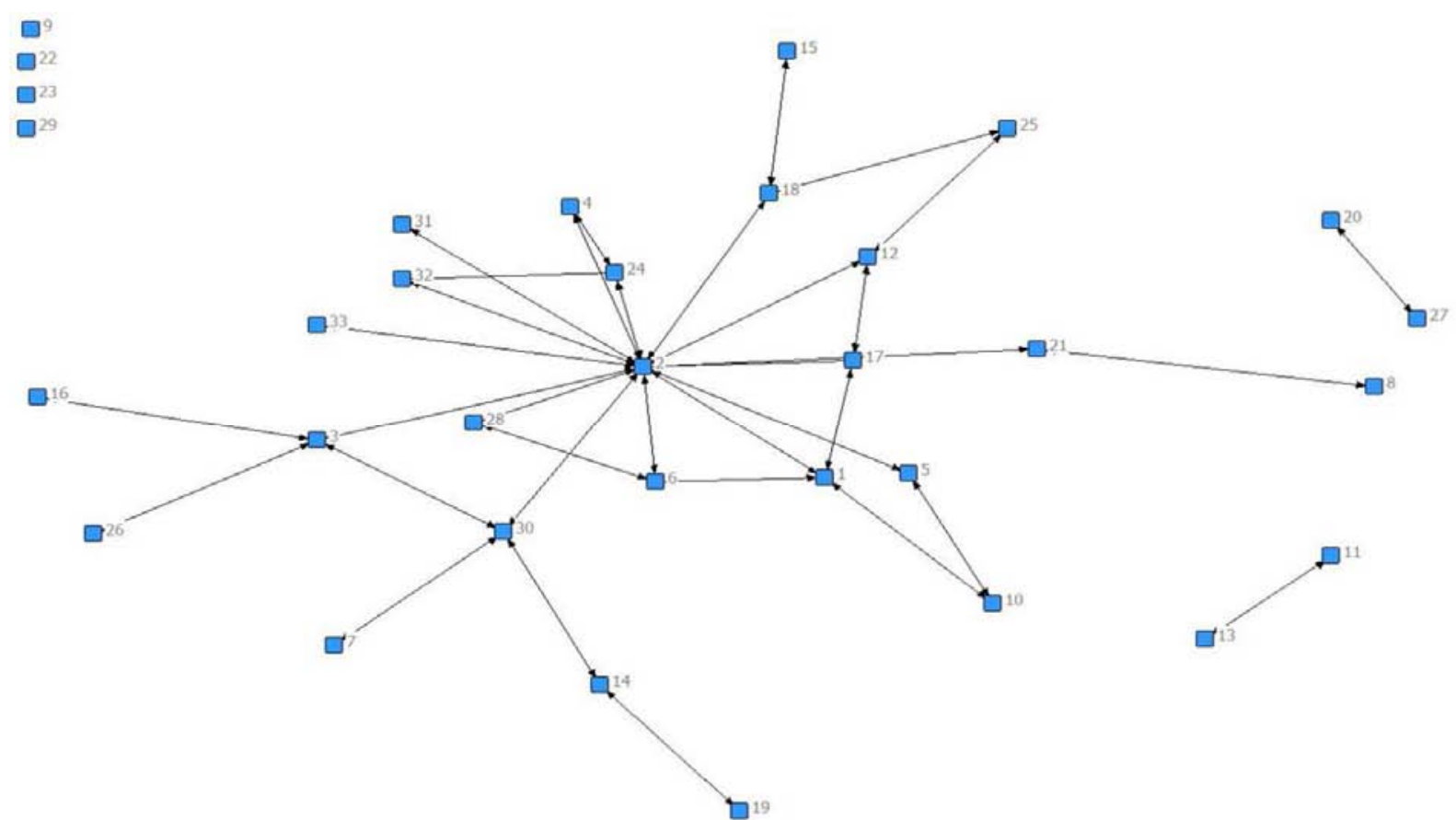

图2 小木虫找工作论坛会员的结点中心度可视化. 
通过将表3、图2中和表1对比可以清晰地看出, 小木 虫找工作论坛中的权力集中度与发帖数量不一定成正比, 如中心度第一的 2 号会员发帖量是第二, 而中心度第四的 30 号会员发帖量才 5 个位于并列 30 名。

(2) 中间中心性
中间中心性测量的是行动者对社会网络中行动者对 资源信息的控制程度, 如果一个结点处在越多结点的交通 网络上, 则证明该结点的中间中心度越高。笔者对所分析 的33名会员的中间中心度均做了计算, 如图3所示:

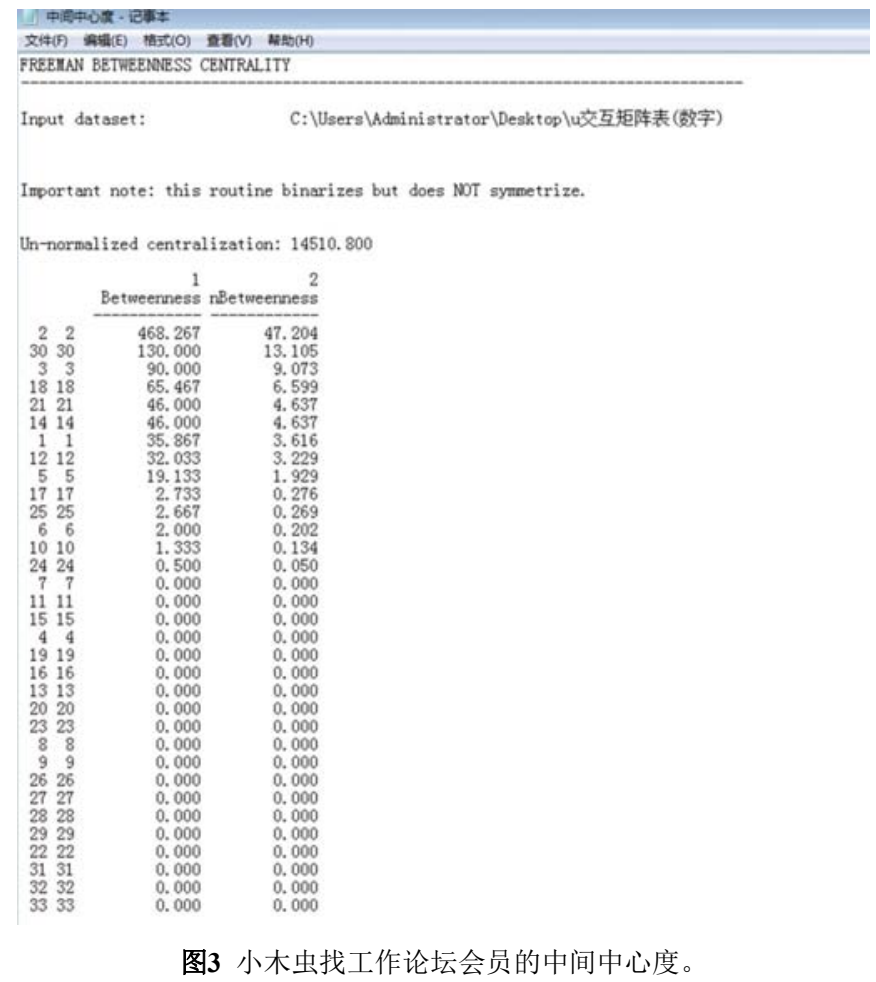

从图 3 可以看出 2 号会员的中间中心度最高, 处于整个 网络交通的中心位置, 30 号和 3 号会员次之。而中间中心 度为 0 的 19 名会员均不在其他的交通网络上。

(3) 接近中心性（整体中心性）
考察一个点传播信息时不靠其他节点的程度。如果一 个点离网络中其他所有点的距离都很短, 说明该点具有较 高的接近中心度，不容易受制于其他结点。将小木虫找工 作论坛中的成员接近中心性分析后得到如图4所示结果。

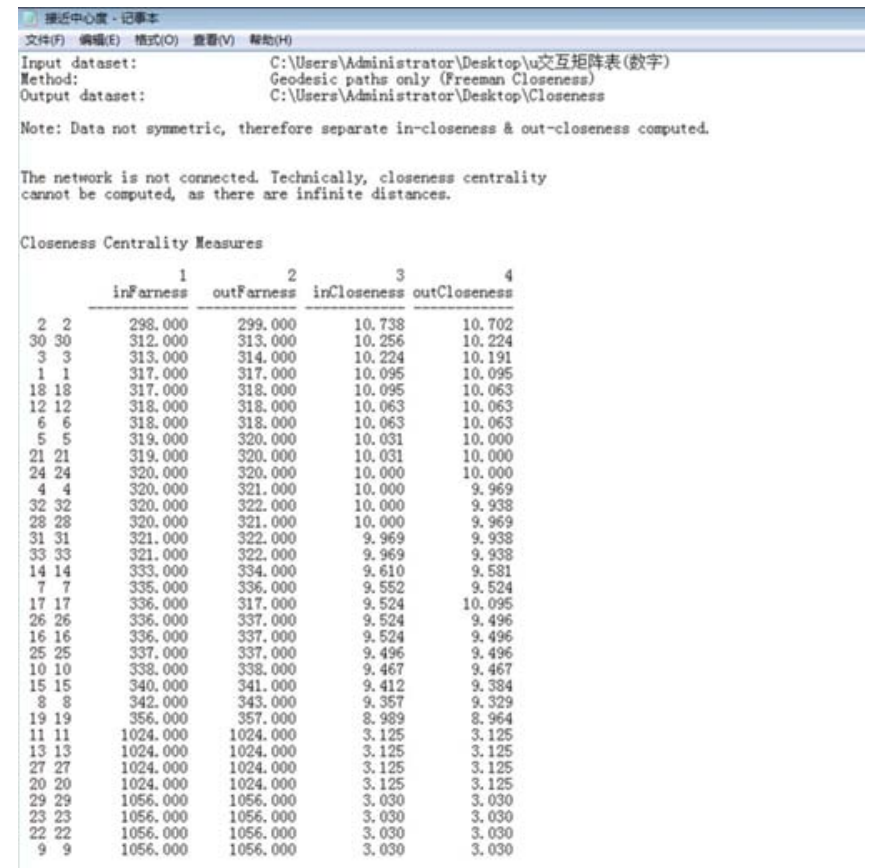

图4 小木虫找工作论坛会员的接近中心度。 
将图 3 的中间中心度和图4的接近中心度对比可知两 者基本成正比，之间排名基本相同不会相差很大。

(4) 凝聚子群分析
群体是指具有特定联系的一些人的组合，他们之间互 动频繁, 而凝聚力则反映了群体的团结性。小木虫找工作 论坛34名成员的子群分布状况如图5所示:

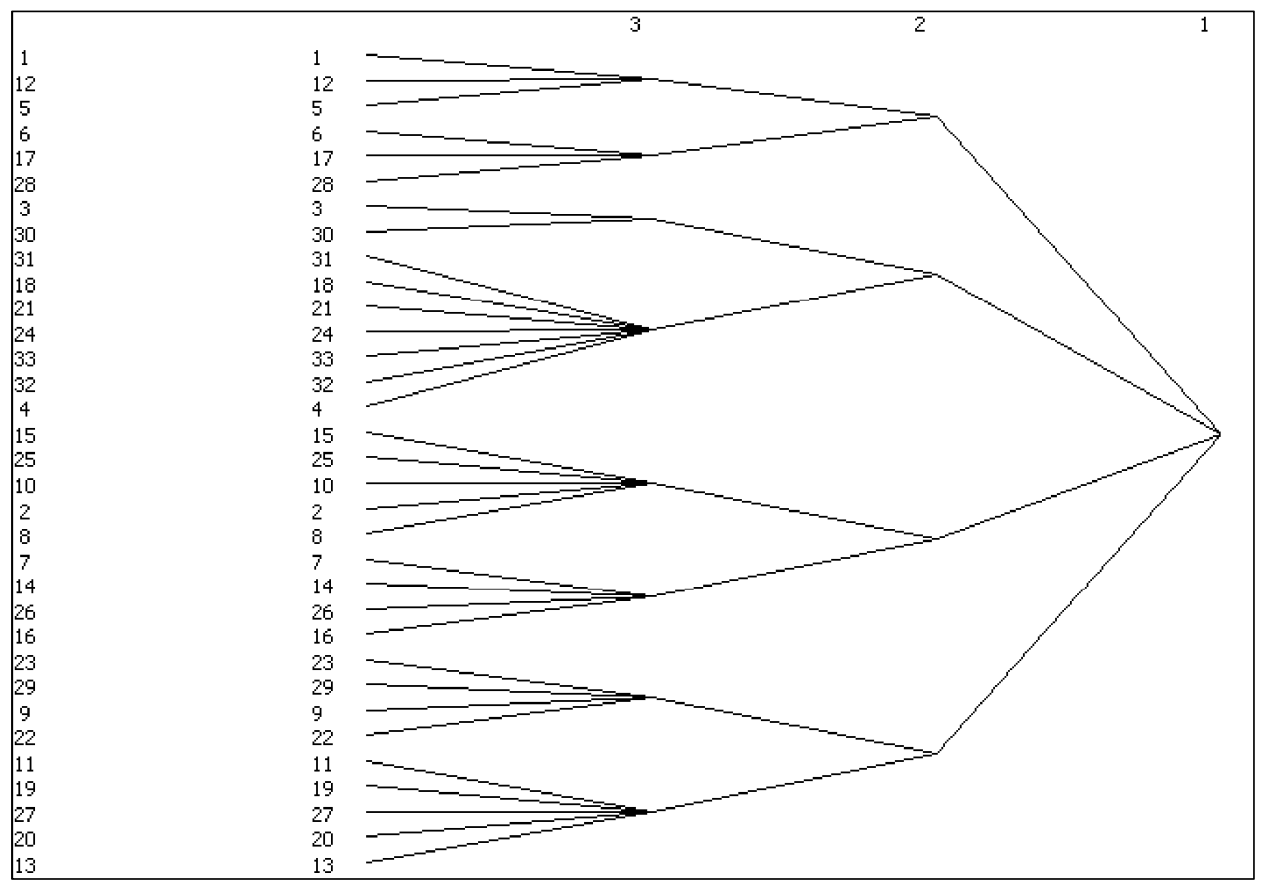

图5 小木虫找工作论坛会员凝聚子群分析。

\section{4. 小木虫找工作论坛交流特点分析}

根据前面所有关于小木虫找工作论坛的各类数据和 特性的分析, 我们可以得到以下几个结论:

（1）小木虫找工作论坛整体交流密度低仅为 0.0644 , 密 度低说明整个论坛的交流网络存在一些问题，即论 坛虽然成员和总贴数很多但是成员间交流很分散, 暂时并未形成一个比较集中的交流网络。

为解决此问题可以挖掘论坛主体的高级用户, 其发帖 数量较大, 影响力较强。这一传播过程启示我们, 如果能 从帖子点击数(或回复数)所形成的时间序列进行网络论坛
话题的热度趋势预测, 判断哪些帖子是热帖, 则可以根据 预测挖掘潜在的高级用户, 进而通过对高级用户的分析实 现对网络论坛的管理和分析 [9]。

（2）整体中心性较高为42.84（图6），说明论坛集权较 为集中，成员之间交流不够充分。造成这个现象的 主要原因是，大部分会员来小木虫找工作论坛时发 帖或者回帖后并没有后续持续进行帖子的追踪, 更 没有对别人的问题或者解答进行相应的记录和回答。 2 号和 30 号会员都是论坛版主, 权利相对集中。

\section{DESCRIPTIVE STATISTICS}

$\begin{array}{lrrrr} & & 1 & 2 & 3 \\ & & \text { Degree } & \text { NrmDegree } & \text { Share } \\ 1 & \text { Mean } & 2.121 & 6.629 & 0.030 \\ 2 & \text { Std Dev } & 2.544 & 7.949 & 0.036 \\ 3 & \text { Sum } & 70.000 & 218.750 & 1.000 \\ 4 & \text { Variance } & 6.470 & 63.185 & 0.001 \\ 5 & \text { SSQ } & 362.000 & 3535.156 & 0.074 \\ 6 & \text { MCSSQ } & 213.515 & 2085.109 & 0.044 \\ 7 & \text { Euc Norm } & 19.026 & 59.457 & 0.272 \\ 8 & \text { Minimum } & 0.000 & 0.000 & 0.000 \\ 9 & \text { Maximum } & 15.000 & 46.875 & 0.214\end{array}$

Network Centralization $=42.84 \%$

Heterogeneity $=7.39 \%$. Normalized $=4.49 \%$

Actor-by-centrality matrix saved as dataset FreemanDegree

图6 小木虫找工作论坛整体中心性分析。 
（3）群体中介性

小木虫找工作论坛群体中介性较高为45.71（图7）, 说明该论坛虽然是对外公开开放的但是仍然存在交流信 息被少数人垄断的可能性存在, 就像 2 号和 30 号会员是版 主，很大程度地控制了整个论坛的交流情况。
知识交流是一种沟通与互动的过程, 含有重建的行为, 包括学习、共享他人的知识, 通过这样的过程实现知识集 成和再创造[10]。因此, 在非正式学术交流的过程中尽量 实现知识的共享和再创造, 避免出现垄断的现象。

\section{DESCRIPTIVE STATISTICS FOR EACH MEASURE}

\begin{tabular}{|c|c|c|}
\hline & $\begin{array}{r}1 \\
\text { Betweenness }\end{array}$ & $\begin{array}{r}2 \\
\text { nBetweenness }\end{array}$ \\
\hline Mean & & \\
\hline Std Dev & & \\
\hline Sum & 942 & 94.960 \\
\hline 4 Variance & 6927 & 395 \\
\hline SSQ & 25549 & 259 \\
\hline MCSSQ & 2286 & 232 \\
\hline Euc Norm & 505 & 50. \\
\hline Minim & & \\
\hline Maximum & 468.267 & 47.204 \\
\hline
\end{tabular}

Network Centralization Index $=45.71 \%$

Output actor-by-centrality measure matrix saved as dataset FreemanBetweenness

图7 小木虫找工作论坛群体中介性分析。

（4）论坛初步形成 8 个小子群和 4 个大子群, 4 个小团体之 间有桥梁连接, 这一方面说明小木虫生命科学论坛 的交流有逐渐深化的趋势, 另一方面也说明, 小木 虫的交流渠道较为顺畅, 小团体间不存在隔断。

(5) 成员及交互分析

笔者将成员发帖量、中间中心性和结点中心性三个特 性进行了排序, 现取前四个做出了对比如表4所示:

表4 小木虫找工作论坛成员对比分析。

\begin{tabular}{llll}
\hline 排序 & 发帖量 & 中间中心性 & 结点中心性 \\
\hline 1 & 1 & 2 & 2 \\
2 & 2 & 30 & 1 \\
3 & 3 & 3 & 3 \\
4 & 4 & 18 & 30 \\
\hline
\end{tabular}

从表4中可以看出，30号成员虽然发帖量很少但是中 间中心性和结点中心性都排前四名, 说明对整个论坛的控 制力较高。3 号会员一般在论坛中都是发的求助帖, 在选 取的 5000 个帖子中每次发表的帖子参与人数都很多, 引起 了大家的共鸣和交流。1 号会员发帖数最多，但是几乎全 部发的是有关招聘的帖子, 所以与大家的交互不够多因此 他的中间中心度不高。

\section{5. 结语}

本论文依托小木虫找工作论坛针对目前非正式学术 交流现状和特点进行了分析, 通过对模态和层次、密度、 中心性等分析深刻剖析了现阶段非正式学术交流人员的 交互行为特点以及非学术交流的多方面特征, 以期对非学 术交流提供参考和建议。但不可否认的是, 想推动整个非 正式学术交流的氛围和效果是一个长期的过程。因此, 需
要在时间中对这一交流过程进行不断探索和发展, 并从实 践中汲取经验, 推动该研究逐步走向深入。

\section{参考文献}

[1] 方卿. 论网络载体环境下科学信息交流过程的基本特征. 情 报理论与实践, 2002,23(2):95-98,114。

[2] 刘佳. 基于网络的学术信息交流方法与模式研究: [学位论 文]. 吉林: 吉林大学, 2007。

[3] 克兰. 无形学院. 北京: 华夏出版社, 1988.2-31。

[4] 方卿. 论网络载体环境下科学信息交流过程的基本特征. 情 报理论与实践, 2002,25(4):852-862。

[5] 屈宝强. 网络学术论坛中的科研合作行为及其反思一以 “小生”学术论坛为例 $[\mathrm{J}]$. 科技管理研究, 2010,30(10):215-218。

[6] 郑璐. 论坛虚拟学习社区的知识交流模式一以“小木虫论 坛”为例 [J]. 高校图书情报论坛, 2014,13(01):37-39+52。

[7] 毛波, 尤雯雯. 虚拟社区成员分类模型 $[J]$. 清华大学学报(自 然科学版), 2006:1069-1073。

[8] 刘军, 《整体网分析讲义—UCINET软件应用》, 第二 界社会网与关系管理研讨会资料, 哈尔滨: 哈尔滨工程大 学社会学系, 2017年1月18日。

[9] 张虹. 基于数据挖掘的网络论坛话题热度趋势预报 $[\mathrm{J}]$. 计算 机工程与应用, 2007, 43(31):159-162。

[10] 方芳，周树英．高校多校区图书馆文献资源共享探析以九江学院图书馆为例 [J].河北科技图苑, 2012(5):57-59。 